\title{
Axiomatic systems and topological semantics for intuitionistic temporal logic ${ }^{\star}$
}

\author{
Joseph Boudou ${ }^{1}$, Martín Diéguez ${ }^{20000-0003-3440-4348]}$, David \\ Fernández-Duque ${ }^{3[0000-0001-8604-4183]}$, and Fabián \\ Romero 1 [0000-0003-1242-5391] \\ 1 IRIT, Toulouse University, Toulouse, France. \\ \{Joseph.Boudou, Fabian.Romero\}@irit.fr \\ 2 LAB-STICC, CERV, ENIB. Brest, France. \\ martin.dieguez@enib.fr \\ 3 Department of Mathematics, Ghent University. Ghent, Belgium. \\ David.FernandezDuque@UGent. be
}

\begin{abstract}
The importance of intuitionistic temporal logics in Computer Science and Artificial Intelligence has become increasingly clear in the last few years. From the proof-theory point of view, intuitionistic temporal logics have made it possible to extend functional languages with new features via type theory, while from its semantical perspective several logics for reasoning about dynamical systems and several semantics for logic programming have their roots in this framework. In this paper we propose four axiomatic systems for intuitionistic linear temporal logic and show that each of these systems is sound for a class of structures based either on Kripke frames or on dynamic topological systems. Our topological semantics features a new interpretation for the 'henceforth' modality that is a natural intuitionistic variant of the classical one. Using the soundness results, we show that the four logics obtained from the axiomatic systems are distinct.
\end{abstract}

\section{Introduction}

Intuitionistic logic (IL) 24 enjoys a myriad of interpretations based on computation, information or topology, making it a natural framework to reason about dynamic processes in which these phenomena play a crucial role. Thus it should not be surprising that combinations of intuitionistic logic and linear temporal logic (LTL) 27] have been proposed for applications within several different contexts.

The first involves the Curry-Howard correspondence [17, which identifies intuitionistic proofs with the $\lambda$-terms of functional programming. Several extensions of the $\lambda$-calculus with operators from LTL have been proposed in order to introduce new features to functional languages: Davies [78] has suggested

* Martín Diéguez is funded by the ANR-12-ASTR-0020 project STRATEGIC and the European COST Action CA17124. 
adding a 'next' (o) operator to IL in order to define the type system $\lambda^{\circ}$, which allows extending functional languages with staged computation ${ }^{4}[13$. Davies and Pfenning [9] proposed the functional language Mini-ML ${ }^{\square}$ which is supported by intuitionistic S4 and allows capturing complex forms of staged computation as well as runtime code generation. Yuse and Igarashi later extended $\lambda^{\circ}$ to $\lambda^{\square}[29]$ by incorporating the 'henceforth' operator $(\square)$, useful for modelling persistent code that can be executed at any subsequent state.

Alternately, intuitionistic temporal logics have been proposed as a tool for modelling semantically-given processes. Maier 23. observed that an intuitionistic temporal logic with 'henceforth' and 'eventually' $(\diamond)$ could be used for reasoning about safety and liveness conditions in possibly-terminating reactive systems, and Fernández-Duque 14 has suggested that a logic with 'eventually' can be used to provide a decidable framework in which to reason about topological dynamics. In the areas of nonmonotonic reasoning, knowledge representation (KR), and artificial intelligence, intuitionistic and intermediate logics have played an important role within the successful answer set programming (ASP) [5] paradigm for practical KR, leading to several extensions of modal ASP [6] that are supported by intuitionistic-based modal logics like temporal here and there 3$]$.

Despite interest in the above applications, there is a large gap to be filled regarding our understanding of the computational behaviour of intuitionistic temporal logics. We have successfuly employed semantical methods to show the decidability of the logic ITL $^{\mathrm{e}}$ defined by a natural class of Kripke frames 4] and shown that these semantics correspond to a natural calculus over the $\square$-free fragment [12]. However, as we will see, in the presence of $\square$, new validities arise which may be undesirable from the point of view of an extended Curry-Howard isomorphism. Thus our goal is to provide semantics for weaker axiomatically-defined intuitionistic temporal logics in order to provide tools for understanding their computational behaviour. We demonstrate the power of our semantics by separating several natural axiomatically-given calculi, which in particular answers in the negative a conjecture of Yuse and Igarashi [29] that the Gentzen-style and the Hilbert-style calculi presented there prove the same set of formulas.

There have already been some notable efforts towards a semantical study of intuitionistic temporal logics. Kojima and Igarashi [19 endowed Davies's logic with Kripke semantics and provided a complete deductive system. Bounded-time versions of logics with henceforth were later studied by Kamide and Wansing [18. Both use semantics based on Simpson's bi-relational models for intuitionistic modal logic [28. Since then, Balbiani and the authors have shown that temporal here-and-there is decidable and enjoys a natural axiomatization [3]. Topological semantics for intuitionistic modal and tense logics have also been studied by Davoren et al. 10[11, and Kremer suggested a topologically-defined intuitionistic variant of LTL with $\circ$ and $\square$ 21]. The decidability of Kremer's

\footnotetext{
${ }^{4}$ Staged computation is a technique that allows dividing the computation in order to exploit the early availability of some arguments.
} 
logic remains open, but Fernández-Duque has shown that a similar logic with 'eventually' $\diamond$ instead of $\square$ is decidable [14.

In this paper we lay the groundwork for an axiomatic treatment of intuitionistic linear temporal logics. We will introduce a 'minimal' intuitionistic temporal logic, $\mathrm{ITL}^{0}$, defined by adding standard axioms of LTL to intuitionistic modal logic. We also consider additional Fischer Servi axioms and a 'constant domain' axiom $\square(p \vee q) \rightarrow \square p \vee \diamond q$. Combining these, we obtain four intuitionistic temporal logics, each of them sound for a class of structures: the two logics with the constant domain axiom are sound for the class of dynamic posets, and the Fischer Servi axioms correspond to backwards-confluence of the transition function.

The constant domain axiom is not derivable from the others, and to show this, we will consider topological semantics for intuitionistic temporal logic. As our axioms involve both $\diamond$ and $\square$, we would like to be able to interpret both tenses. Kremer 21] observed that his semantics for $\square$ do not satisfy some key LTL validities, namely $\square p \rightarrow \circ \square p, \square \circ p \rightarrow \circ \square p$, and $\square p \rightarrow \square \square p$. Consequently $\mathrm{ITL}^{0}$ is not sound for this interpretation. In order to obtain models of $\mathrm{ITL}^{0}$, we propose an alternative interpretation for $\square$. Our approach is natural from an algebraic perspective, as we define the interpretation of $\square \varphi$ via a greatest fixed point in the Heyting algebra of open sets. We will show that dynamic topological systems provide semantics for the logics without the constant domain axiom, from which we conclude the independence of the latter. Moreover, we show that the Fischer Servi axioms are valid for the class of open dynamical topological systems. The constant domain axiom shows that the $\{\diamond, \square\}$-logic of dynamic posets is different from that of dynamic topological systems. We show via an alternative axiom that the $\{0, \square\}$-logics are also different.

LAyOuT. Section 2 introduces the syntax and the four axiomatic systems we propose for intuitionistic temporal logic. Section 3 reviews dynamic topological systems, which are used in Section 4 to provide semantics for our formal language. Section 5 shows that each of the four logics is sound for a class of dynamical systems. These soundness results are used in Section 6 to show that the four logics are pairwise distinct. Finally, Section 7 lists some open questions.

\section{Syntax and axiomatics}

In this section we will introduce four natural intuitionistic temporal logics. All of the axioms have appeared either in the intuitionistic logic, the temporal logic, or the intuitionistic modal logic literature. They will be based on the language of linear temporal logic, as defined next.

Fix a countably infinite set $\mathbb{P}$ of propositional variables. The language $\mathcal{L}$ of intuitionistic (linear) temporal logic ITL is given by the grammar

$$
\perp|p| \varphi \wedge \psi|\varphi \vee \psi| \varphi \rightarrow \psi|\circ \varphi| \nabla \varphi \mid \square \varphi,
$$

where $p \in \mathbb{P}$. As usual, we use $\neg \varphi$ as a shorthand for $\varphi \rightarrow \perp$ and $\varphi \leftrightarrow \psi$ as a shorthand for $(\varphi \rightarrow \psi) \wedge(\psi \rightarrow \varphi)$. We read $\circ$ as 'next', $\diamond$ as 'eventually', 
and $\square$ as 'henceforth'. Given any formula $\varphi$, we denote the set of subformulas of $\varphi$ by $\operatorname{sub}(\varphi)$. The language $\mathcal{L}_{\diamond}$ is defined as the sublanguage of $\mathcal{L}$ without the modality $\square$. Similarly, $\mathcal{L}_{\square}$ is the language without $\diamond$.

We begin by establishing our basic axiomatization. It is obtained by adapting the standard axioms and inference rules of LTL 22, as well as their dual versions. To be precise, the logic $\mathrm{ITL}^{0}$ is the least set of $\mathcal{L}$-formulas closed under the following rules and axioms.

i) All intuitionistic tautologies.

ii) $\neg \circ \perp$

iii) $\circ(\varphi \wedge \psi) \leftrightarrow(\circ \varphi \wedge \circ \psi)$;

iv) $\circ(\varphi \vee \psi) \leftrightarrow(\circ \varphi \vee \circ \psi)$;

v) $\circ(\varphi \rightarrow \psi) \rightarrow(\circ \varphi \rightarrow \circ \psi)$;

vi) $\square(\varphi \rightarrow \psi) \rightarrow(\square \varphi \rightarrow \square \psi)$;

vii) $\square(\varphi \rightarrow \psi) \rightarrow(\diamond \varphi \rightarrow \diamond \psi)$; viii) $\square \varphi \rightarrow \varphi \wedge \circ \square \varphi$;

ix) $\varphi \vee \circ \diamond \varphi \rightarrow \diamond \varphi$

x) $\square(\varphi \rightarrow \circ \varphi) \rightarrow(\varphi \rightarrow \square \varphi)$;

xi) $\square(\circ \varphi \rightarrow \varphi) \rightarrow(\diamond \varphi \rightarrow \varphi)$;

xii) $\frac{\varphi \varphi \rightarrow \psi}{\psi}$;

xiii) $\frac{\varphi}{\circ \varphi}$.

Each axiom is either included in the axiomatization of Goldblatt [16, page 87] or is a mild variant of one of them (e.g., a contrapositive); this is standard in intuitionistic modal logic, as such variants are needed to account for the independence of the basic connectives. We do not consider 'until' in this paper, but have studied its intuitionistic semantics in 2. Modal intuitionistic logics often involve additional axioms, and in particular Fischer Servi [15] includes the schema

$$
\mathrm{FS}_{\diamond}(\varphi, \psi) \stackrel{\text { def }}{=}(\diamond \varphi \rightarrow \square \psi) \rightarrow \square(\varphi \rightarrow \psi) .
$$

Recalling that $\circ$ is self-dual, we also define

$$
\mathrm{FS}_{\circ}(\varphi, \psi) \stackrel{\text { def }}{=}(\circ \varphi \rightarrow \circ \psi) \rightarrow \circ(\varphi \rightarrow \psi) .
$$

Later we will show that these schemas lead to strictly stronger logics. Finally, we consider additional axioms reminiscent of constant domain axioms in firstorder intuitionistic logic. As we will see, in the context of intuitionistic temporal logics, these axioms separate Kripke semantics from the more general topological semantics.

$$
\begin{aligned}
\mathrm{CD}(\varphi, \psi) & \stackrel{\text { def }}{=} \square(\varphi \vee \psi) \rightarrow \square \varphi \vee \diamond \psi \\
\mathrm{BI}(\varphi, \psi) & \stackrel{\text { def }}{=} \square(\varphi \vee \psi) \wedge \square(\circ \psi \rightarrow \psi) \rightarrow \square \varphi \vee \psi .
\end{aligned}
$$

Here, CD stands for 'constant domain' and BI for 'backward induction'.

From a constructive perspective CD might not be desirable, as from $\square(\varphi \vee \psi)$ one cannot in general extract an upper bound for a witness for $\diamond \psi 5$ The axiom $\mathrm{BI}$ is meant to be a $\diamond$-free approximation to $\mathrm{CD}$, as witnessed by the following.

\footnotetext{
${ }^{5}$ For example, if $\varphi$ represents the 'active' states and $\psi$ the 'halting' states of a program, then CD would require us to decide whether the program halts, which is not possible to do constructively.
} 
Proposition 1. $\mathrm{ITL}^{0} \vdash \mathrm{CD}(p, q) \rightarrow \mathrm{BI}(p, q)$.

Proof. We reason within $\mathrm{ITL}^{0}$. Assume that 1) $\left.\mathrm{CD}(p, q), 2\right) \square(\circ q \rightarrow q)$, and 3) $\square(p \vee q)$. From 1) and 3) we obtain $\square p \vee \diamond q$, which together with 2) and axiom xi) gives us $\square p \vee q$, as needed.

With this, we define the following logics:

$$
\mathrm{ITL}^{\mathrm{FS}} \equiv \mathrm{ITL}^{0}+\mathrm{FS}_{\diamond}+\mathrm{FS}_{\diamond}, \quad \mathrm{ITL}^{\mathrm{CD}} \equiv \mathrm{ITL}^{0}+\mathrm{CD}, \quad \mathrm{ITL}^{1} \equiv \mathrm{ITL} \mathrm{FS}^{\mathrm{FS}}+\mathrm{ITL}^{\mathrm{CD}} .
$$

We are also interested in logics over sublanguages of $\mathcal{L}$. For any logic $\Lambda$ defined above, let $\Lambda_{\square}$ be defined by restricting similarly all rules and axioms to $\mathcal{L}_{\square}$, except that when CD is an axiom of $\Lambda$, we add the axiom BI to $\Lambda_{\square}$. The logic $\mathrm{ITL}_{\square}^{0}$ is similar to a Hilbert calculus for the $\wedge, \vee$-free fragment considered by Yuse and Igarashi [29], although they do not include induction but include the axioms $\square \varphi \rightarrow \square \square \varphi$ and $\square \circ \varphi \leftrightarrow \circ \square \varphi$. It is not difficult to check that the latter are derivable from our basic axioms, and hence their logic is contained in $\operatorname{ITL}_{\square}^{0}$.

We also define $\Lambda_{\diamond}$ be the logic obtained by restricting all rules and axioms to $\mathcal{L}_{\diamond}$, and adding the rules $\frac{\varphi \rightarrow \psi}{\diamond \varphi \rightarrow \diamond \psi}$ and $\frac{\circ \varphi \rightarrow \varphi}{\diamond \varphi \rightarrow \varphi}$. Note that these rules correspond to axioms vii), xi) respectively, but do not involve $\square$. In this paper we are mostly concerned with logics including 'henceforth', but $\square$-free logics are studied in detail in 12 .

\section{Dynamic topological systems}

The four logics over $\mathcal{L}$ defined above are pairwise distinct. We will show this by introducing semantics for each of them. They will be based on dynamic topological systems (or dynamical systems for short), which, as was observed in 14, generalize their Kripke semantics 4. Let us first recall the definition of a topological space [25]:

Definition 1. A topological space is a pair $(X, \mathcal{T})$, where $X$ is a set and $\mathcal{T}$ a family of subsets of $X$ satisfying a) $\varnothing, X \in \mathcal{T}$; b) if $U, V \in \mathcal{T}$ then $U \cap V \in \mathcal{T}$, and c) if $\mathcal{O} \subseteq \mathcal{T}$ then $\bigcup \mathcal{O} \in \mathcal{T}$. The elements of $\mathcal{T}$ are called open sets.

If $x \in X$, a neighbourhood of $x$ is an open set $U \subseteq X$ such that $x \in U$. Given a set $A \subseteq X$, its interior, denoted $A^{\circ}$, is the largest open set contained in $A$. It is defined formally by

$$
A^{\circ}=\bigcup\{U \in \mathcal{T}: U \subseteq A\} .
$$

Dually, we define the closure $\bar{A}$ as $X \backslash(X \backslash A)^{\circ}$; this is the smallest closed set containing $A$.

If $(X, \mathcal{T})$ is a topological space, a function $S: X \rightarrow X$ is continuous if, whenever $U \subseteq X$ is open, it follows that $S^{-1}[U]$ is open. The function $S$ is open if, whenever $V \subseteq X$ is open, then so is $S[V]$. An open, continuous function is an interior map, and a bijective interior map is a homeomorphism.

A dynamical system is then a topological space equipped with a continuous function: 
Definition 2. A dynamical (topological) system is a triple $\mathcal{X}=(X, \mathcal{T}, S)$ such that $(X, \mathcal{T})$ is a topological space and $S: X \rightarrow X$ is continuous. We say that $\mathcal{X}$ is invertible if $S$ is a homeomorphism, i.e., $S$ is bijective and $S^{-1}$ is also a continuous function, and open if $S$ is an interior map.

Topological spaces generalize posets in the following way. Let $\mathcal{F}=(W, \preccurlyeq)$ be a poset; that is, $W$ is any set and $\preccurlyeq$ is a transitive, reflexive, antisymmetric relation on $W$. To see $\mathcal{F}$ as a topological space, define $\uparrow w=\{v: w \preccurlyeq v\}$. Then consider the topology $\mathcal{T}_{\preccurlyeq}$ on $W$ given by setting $U \subseteq W$ to be open if and only if, whenever $w \in U$, we have $\uparrow w \subseteq U$. A topology of this form is a up-set topology [1. The interior operator on such a topological space can be computed by

$$
A^{\circ}=\{w \in W: \uparrow w \subseteq A\} ;
$$

i.e., $w$ lies on the interior of $A$ if whenever $v \succcurlyeq w$, it follows that $v \in A$.

Throughout this text we will often identify partial orders with their corresponding topologies, and many times do so tacitly. In particular, a dynamical system generated by a poset is called a dynamic poset. It will be useful to characterize the continuous and open functions on posets:

Lemma 1. Consider a poset $(W, \preccurlyeq)$ and a function $S: W \rightarrow W$. Then,

1. $S$ is continuous with respect to the up-set topology if and only if, whenever $w \preccurlyeq w^{\prime}$, it follows that $S(w) \preccurlyeq S\left(w^{\prime}\right)$, and

2. $S$ is open with respect to the up-set topology if whenever $S(w) \preccurlyeq v$, there is $w^{\prime} \in W$ such that $w \preccurlyeq w^{\prime}$ and $S\left(w^{\prime}\right)=v$.

These are confluence properties common in multi-modal logics; open, continuous maps on a poset are called persistent.

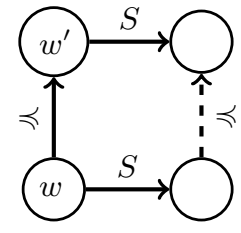

(a) Continuity

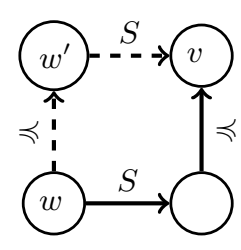

(b) Openness

Fig. 1: On a dynamic poset the above diagrams can always be completed if $S$ is continuous or open, respectively.

\section{Semantics}

In this section we will see how dynamical systems can be used to provide a natural intuitionistic semantics for the language of linear temporal logic. 
Formulas are interpreted as open subspaces of a dynamical system. Each propositional variable $p$ is assigned an open set $\llbracket p \rrbracket$, and then $\llbracket \cdot \rrbracket$ is defined recursively for more complex formulas according to the following:

Definition 3. Given a dynamical system $\mathcal{X}=(X, \mathcal{T}, S)$, a valuation on $\mathcal{X}$ is a function $\llbracket \cdot \rrbracket: \mathcal{L} \rightarrow \mathcal{T}$ such that:

$$
\begin{array}{ll}
\llbracket \perp \rrbracket=\varnothing & \llbracket \circ \varphi \rrbracket=S^{-1} \llbracket \varphi \rrbracket \\
\llbracket \varphi \wedge \psi \rrbracket=\llbracket \varphi \rrbracket \cap \llbracket \psi \rrbracket & \llbracket \diamond \varphi \rrbracket=\bigcup_{n \geq 0} S^{-n} \llbracket \varphi \rrbracket \\
\llbracket \varphi \vee \psi \rrbracket=\llbracket \varphi \rrbracket \cup \llbracket \psi \rrbracket & \\
\llbracket \varphi \rightarrow \psi \rrbracket=((X \backslash \llbracket \varphi \rrbracket) \cup \llbracket \psi \rrbracket)^{\circ} & \llbracket \square \varphi \rrbracket=\bigcup\{U \in \mathcal{T}: S[U] \subseteq U \subseteq \llbracket \varphi \rrbracket\}
\end{array}
$$

A tuple $\mathcal{M}=(X, \mathcal{T}, S, \llbracket \cdot \rrbracket)$ consisting of a dynamical system with a valuation is a dynamic topological model, and if $\mathcal{T}$ is generated by a partial order, we will say that $\mathcal{M}$ is a dynamic poset model.

All of the semantic clauses are standard from either intuitionistic or temporal logic, with the exception of that for $\square \varphi$, which we discuss in greater detail below. It is not hard to check by structural induction on $\varphi$ that $\llbracket \varphi \rrbracket$ is uniquely defined given any assignment of the propositional variables to open sets, and that $\llbracket \varphi \rrbracket$ is always open. We define validity in the standard way, and with this introduce four additional semantically-defined logics, two of which were already studied by us in Boudou et al. [4].

Definition 4. If $\mathcal{M}=(X, \mathcal{T}, S, \llbracket \cdot \rrbracket)$ is any dynamic topological model and $\varphi \in \mathcal{L}$ is any formula, we write $\mathcal{M} \models \varphi$ if $\llbracket \varphi \rrbracket=X$. Similarly, if $\mathcal{X}=(X, \mathcal{T}, S)$ is a dynamical system, we write $\mathcal{X} \models \varphi$ if for any valuation $\llbracket \cdot \rrbracket$ on $\mathcal{X}$, we have that $(\mathcal{X}, \llbracket \cdot \rrbracket) \models \varphi$. Finally, if $\Omega$ is a class of structures, we write $\Omega \models \varphi$ if for every $\mathcal{A} \in \Omega, \mathcal{A}=\varphi$, in which case we say that $\varphi$ is valid on $\Omega$.

We denote the set of formulas valid over the class of all dynamical systems by $\mathrm{ITL}^{\mathrm{c}}$, over the class of all dynamic posets by $\mathrm{ITL}^{\mathrm{e}}$, over the class of all persistent posets by $\mathrm{ITL}^{\mathrm{P}}$ and over the class of all open dynamical systems by $\mathrm{ITL}^{\circ}$. If $\Lambda$ is one of these four logics we define $\Lambda_{\square}=\Lambda \cap \mathcal{L}_{\square}$ and $\Lambda_{\diamond}=\Lambda \cap \mathcal{L}_{\diamond}$.

In practice, it is convenient to have a 'pointwise' characterization of the semantic clauses of Definition 3. For a model $\mathcal{M}=(X, \mathcal{T}, S, \llbracket \cdot \rrbracket), x \in X$ and $\varphi \in \mathcal{L}$, we write $\mathcal{M}, x \models \varphi$ if $x \in \llbracket \varphi \rrbracket$, and $\mathcal{M}=\varphi$ if $\llbracket \varphi \rrbracket=X$. Then, in view of (1), given formulas $\varphi$ and $\psi, \mathcal{M}, x \models \varphi \rightarrow \psi$ if and only if there is a neighbourhood $U$ of $x$ such that for all $y \in U$, if $\mathcal{M}, y \models \varphi$ then $\mathcal{M}, y \models \psi$; note that this is a special case of neighbourhood semantics [26].

Using (2), this can be simplified somewhat in the case that $\mathcal{T}$ is generated by a partial order $\preccurlyeq$ :

Proposition 2. If $(X, \preccurlyeq, S, \llbracket \cdot \rrbracket)$ is a dynamic poset model, $x \in X$, and $\varphi, \psi$ are formulas, then $\mathcal{M}, x \models \varphi \rightarrow \psi$ if and only if whenever $y \succcurlyeq x$ and $\mathcal{M}, y \models \varphi$, it follows that $\mathcal{M}, y \models \psi$. 
This is the standard relational interpretation of implication, and thus topological semantics are a generalization of the usual Kripke semantics. Now let us discuss the topological interpretation of 'henceforth', which is the main novelty in our semantics. In classical temporal logic, $\llbracket \square \varphi \rrbracket$ is the largest set contained in $\llbracket \varphi \rrbracket$ which is closed under $S$. In our semantics, $\llbracket \square \varphi \rrbracket$ is the greatest open set which is closed under $S$. From this perspective, our interpretation is the natural intuitionistic variant of the classical one. If $\mathcal{M}, x \models \square \varphi$, this fact is witnessed by an open, $S$-invariant neighbourhood of $x$, where $U \subseteq X$ is $S$-invariant if $S[U] \subseteq U$.

Proposition 3. If $(X, \mathcal{T}, S, \llbracket \cdot \rrbracket)$ is a dynamic topological model, $x \in X$, and $\varphi$ is any formula, then $\mathcal{M}, x \models \square \varphi$ if and only if there is an $S$-invariant neighbourhood $U$ of $x$ such that for all $y \in U, \mathcal{M}, y=\varphi$.

In fact, the open, $S$-invariant sets form a topology; that is, the family of $S$-invariant open sets is closed under finite intersections and arbitrary unions. This topology is coarser than $\mathcal{T}$, in the sense that every $S$-invariant open set is (tautologically) open. Thus $\square$ can itself be seen as an interior operator based on a coarsening of $\mathcal{T}$, and $\llbracket \square \varphi \rrbracket$ is always an $S$-invariant open set.

Example 1. As usual, the real number line is denoted by $\mathbb{R}$ and we assume that it is equipped with the standard topology, where $U \subseteq \mathbb{R}$ is open if and only if it is a union of intervals of the form $(a, b)$. Consider a dynamical system based on $\mathbb{R}$ with $S: \mathbb{R} \rightarrow \mathbb{R}$ given by $S(x)=2 x$. We claim that for any model $\mathcal{M}$ based on $(\mathbb{R}, S)$ and any formula $\varphi, \mathcal{M}, 0 \models \square \varphi$ if and only if $\mathcal{M} \models \varphi$.

To see this, note that one implication is obvious since $\mathbb{R}$ is open and $S$ invariant, so if $\llbracket \varphi \rrbracket=\mathbb{R}$ it follows that $\mathcal{M}, 0 \models \square \varphi$. For the other implication, assume that $\mathcal{M}, 0=\square \varphi$, so that there is an $S$-invariant, open $U \subseteq \llbracket \varphi \rrbracket$ with $0 \in U$. It follows from $U$ being open that for some $\varepsilon>0,(-\varepsilon, \varepsilon) \subseteq U$. Now, let $x \in \mathbb{R}$, and let $n$ be large enough so that $\left|2^{-n} x\right|<\varepsilon$. Then, $2^{-n} x \in U$, and since $U$ is $S$-invariant, $x=S^{n}\left(2^{-n} x\right) \in U$. Since $x$ was arbitrary, $U=\mathbb{R}$, and it follows that $\mathcal{M}=\varphi$.

On the other hand, suppose that $0<a<x$ and $(a, \infty) \subseteq \llbracket \varphi \rrbracket$. Then, $(a, \infty)$ is open and $S$-invariant, so it follows that $x \in \llbracket \square \varphi \rrbracket$. Hence in this case we do not require that $\llbracket \varphi \rrbracket=\mathbb{R}$. Similarly, if $x<a<0$ and $(-\infty, a) \subseteq \llbracket \varphi \rrbracket$, we readily obtain $x \in \llbracket \square \varphi \rrbracket$.

As was the case for implication, our interpretation for $\square$ becomes familiar when restricted to Kripke semantics.

Lemma 2. Let $\mathcal{M}=(W, \preccurlyeq, S, \llbracket \cdot \rrbracket)$ be any dynamic poset model, $w \in W$ and $\varphi \in \mathcal{L}$. Then, the following are equivalent:

a) $\mathcal{M}, w \models \square \varphi$; b) $w \in\left(\bigcap_{n<\omega} S^{-n} \llbracket \varphi \rrbracket\right)^{\circ}$; c) for all $n<\omega, \mathcal{M}, S^{n}(w) \models \varphi$.

Proof. First we prove that a) implies b) Assume that $\mathcal{M}, w \models \square \varphi$, so that there is an $S$-invariant neighbourhood $U$ of $w$ with $U \subseteq \llbracket \varphi \rrbracket$. To see that $w \in$ $\left(\bigcap_{n<\omega} S^{-n} \llbracket \varphi \rrbracket\right)^{\circ}$, we must show that if $v \succcurlyeq w$, then $v \in \bigcap_{n<\omega} S^{-n} \llbracket \varphi \rrbracket$. So fix 
such a $v$ and $n<\omega$. Since $U$ is $S$-invariant, $S^{n}(w) \in U$, and since $S^{n}(v) \succcurlyeq S^{n}(w)$ and $U$ is open, $S^{n}(v) \in U$, as needed. Thus $v \in \bigcap_{n<\omega} S^{-n} \llbracket \varphi \rrbracket$, and since $v \succcurlyeq w$ was arbitrary, b) holds.

That b) implies c) is immediate from

$$
\left(\bigcap_{n<\omega} S^{-n} \llbracket \varphi \rrbracket\right)^{\circ} \subseteq \bigcap_{n<\omega} S^{-n} \llbracket \varphi \rrbracket,
$$

so it remains to show that c) implies a). Suppose that for all $n<\omega, \mathcal{M}, S^{n}(w) \models$ $\varphi$, and let $U=\bigcup_{n<\omega} \uparrow S^{n}(w)$. That the set $U$ is open follows from each $\uparrow S^{n}(w)$ being open and unions of opens being open. If $v \in U$, then $v \succcurlyeq S^{n}(w)$ for some $n<\omega$ and hence by upwards persistence, from $\mathcal{M}, S^{n}(w) \models \varphi$ we obtain $\mathcal{M}, v \models \varphi$; moreover, $S(v) \succcurlyeq S^{n+1}(w)$ so $S(v) \in U$. Since $v \in U$ was arbitrary, we conclude that $U$ is $S$-invariant and $U \subseteq \llbracket \varphi \rrbracket$. Thus $U$ witnesses that $\mathcal{M}, w \models \square \varphi$.

Remark 1. Kremer [21] uses b) as the definition of $\llbracket \square \varphi \rrbracket$. However, as we mentioned in the introduction, even our minimal axiomatic system $I \mathrm{TL}^{0}$ is not sound for such an interpretation over arbitrary dynamical systems.

\section{Soundness}

In this section we will show that the four logics we have considered are sound for semantics based on different classes of dynamic topological systems. First we show that our minimal logic is sound for the class of all dynamical systems. The following simple observation will be useful.

Lemma 3. If $\mathcal{M}=(X, \mathcal{T}, S, \llbracket \cdot \rrbracket)$ is any model and $\varphi, \psi \in \mathcal{L}$, then $\mathcal{M} \models \varphi \rightarrow \psi$ if and only if $\llbracket \varphi \rrbracket \subseteq \llbracket \psi \rrbracket$.

Proof. If $\llbracket \varphi \rrbracket \subseteq \llbracket \psi \rrbracket$ then $(X \backslash \llbracket \varphi \rrbracket) \cup \llbracket \psi \rrbracket=X$, so $\llbracket \varphi \rightarrow \psi \rrbracket=((X \backslash \llbracket \varphi \rrbracket) \cup$ $\llbracket \psi \rrbracket)^{\circ}=X^{\circ}=X$. Otherwise, there is $z \in \llbracket \varphi \rrbracket$ such that $z \notin \llbracket \psi \rrbracket$, so that $z \notin((X \backslash \llbracket \varphi \rrbracket) \cup \llbracket \psi \rrbracket)^{\circ}$, i.e. $z \notin \llbracket \varphi \rightarrow \psi \rrbracket$.

Theorem 1. $\mathrm{ITL}^{0}$ is sound for the class of dynamical systems; that is, $\mathrm{ITL}^{0} \subseteq$ $I T L^{c}$.

Proof. Let $\mathcal{M}=(X, \mathcal{T}, S, \llbracket \cdot \rrbracket)$ be any dynamical topological model; we must check that all the axioms (i) xi) are valid on $\mathcal{M}$ and the rules xii), xiii) preserve validity. Note that all intuitionistic tautologies are valid due to the soundness for topological semantics 24. Many of the other axioms can be checked routinely, so we focus only on those axioms involving the continuity of $S$ or the semantics for $\square$.

v) Suppose that $x \in \llbracket \circ(\varphi \rightarrow \psi) \rrbracket$. Then, $S(x) \in \llbracket \varphi \rightarrow \psi \rrbracket$. Since $S$ is continuous and $\llbracket \varphi \rightarrow \psi \rrbracket$ is open, $U=S^{-1} \llbracket \varphi \rightarrow \psi \rrbracket$ is a neighbourhood of $x$. Then, for $y \in U$, if $y \in \llbracket \circ \varphi \rrbracket$, it follows that $S(y) \in \llbracket \varphi \rrbracket \cap \llbracket \varphi \rightarrow \psi \rrbracket$, so that $S(y) \in \llbracket \psi \rrbracket$ and $y \in \llbracket \circ \psi \rrbracket$. 
Since $y \in U$ was arbitrary, $x \in \llbracket \circ \varphi \rightarrow \circ \psi \rrbracket$, thus $\llbracket \circ(\varphi \rightarrow \psi) \rrbracket \subseteq \llbracket \circ \varphi \rightarrow \circ \psi \rrbracket$, and by Lemma 3 (which we will henceforth use without mention), v) is valid on $\mathcal{M}$.

vi) Suppose that $x \in \llbracket \square(\varphi \rightarrow \psi) \rrbracket$. Then, there is an $S$-invariant neighbourhood $U$ of $x$ such that $U \subseteq \llbracket \varphi \rightarrow \psi \rrbracket$. We claim that if $y \in U \cap \llbracket \square \varphi \rrbracket$ it follows that $y \in \llbracket \square \psi \rrbracket$, from which we obtain $x \in \llbracket \square \varphi \rightarrow \square \psi \rrbracket$, as needed. If $y \in U \cap \llbracket \square \varphi \rrbracket$, let $U^{\prime}$ be an $S$-invariant neighbourhood of $y$ such that $U^{\prime} \subseteq \llbracket \varphi \rrbracket$, and define $V=U \cap U^{\prime}$. Then, the set $V$ is an $S$-invariant neighbourhood of $y$. Moreover, if $z \in V$, then $z \in U \subseteq \llbracket \varphi \rightarrow \psi \rrbracket$, while $z \in U^{\prime} \subseteq \llbracket \varphi \rrbracket$, hence $z \in \llbracket \psi \rrbracket$. It follows that $V \subseteq \llbracket \psi \rrbracket$, and thus $y \in \llbracket \square \psi \rrbracket$, as desired.

vi) Observe that $\llbracket \square(\varphi \rightarrow \psi) \rrbracket$ is an $S$-invariant open subset of $\llbracket \varphi \rightarrow \psi \rrbracket$. Similarly, $\llbracket \square \varphi \rrbracket$ is an $S$-invariant open subset of $\llbracket \varphi \rrbracket$. Let

$$
U=\llbracket \square(\varphi \rightarrow \psi) \rrbracket \cap \llbracket \square \varphi \rrbracket .
$$

Since $U$ is open, it suffices to prove that $U \subseteq \llbracket \square \psi \rrbracket$. Moreover, $U$ is $S$-invariant, therefore it suffices to prove that $U \subseteq \llbracket \psi \rrbracket$, which is direct because $U \subseteq \llbracket \varphi \rightarrow \psi \rrbracket \cap$ $\llbracket \varphi \rrbracket$ and $\llbracket \varphi \rightarrow \psi \rrbracket \subseteq(X \backslash \llbracket \varphi \rrbracket) \cup \llbracket \psi \rrbracket$.

vii) As before, suppose that $x \in \llbracket \square(\varphi \rightarrow \psi) \rrbracket$, and let $U$ be an $S$-invariant neighbourhood of $x$ such that $U \subseteq \llbracket \varphi \rightarrow \psi \rrbracket$. If $y \in U \cap \llbracket \nabla \varphi \rrbracket$, then $S^{n}(y) \in \llbracket \varphi \rrbracket$ for some $n$; since $U$ is $S$-invariant, $S^{n}(y) \in U$, hence $S^{n}(y) \in \llbracket \psi \rrbracket$ and $y \in \llbracket \triangleright \psi \rrbracket$. We conclude that $x \in \llbracket \nabla \varphi \rightarrow \diamond \psi \rrbracket$.

viii) Suppose that $x \in \llbracket \square \varphi \rrbracket$, and let $U \subseteq \llbracket \varphi \rrbracket$ be an $S$-invariant neighbourhood of $x$. Then, $x \in U$, so $x \in \llbracket \varphi \rrbracket$. Moreover, $U$ is also an $S$-invariant neighbourhood of $S(x)$, so $S(x) \in \llbracket \square \varphi \rrbracket$ and thus $x \in \llbracket \circ \square \varphi \rrbracket$. We conclude that $x \in \llbracket \varphi \wedge \circ \square \varphi \rrbracket$.

$\mathrm{x})$ Supppose that $x \in \llbracket \square(\varphi \rightarrow \circ \varphi) \rrbracket$. If $x \in \llbracket \varphi \rrbracket$, then $U=\llbracket \varphi \rrbracket \cap \llbracket \square(\varphi \rightarrow \circ \varphi) \rrbracket$ is open (by the intuitionistic semantics) and $S$-invariant, since if $y \in U$, from $y \in \llbracket \varphi \rightarrow \circ \varphi \rrbracket$ we obtain $S(y) \in \llbracket \varphi \rrbracket$. It follows that $U$ is an $S$-invariant neighbourhood of $x$, so $x \in \llbracket \square \varphi \rrbracket$.

xi) Suppose that $x \in \llbracket \square(\circ \varphi \rightarrow \varphi) \rrbracket \cap \llbracket \diamond \varphi \rrbracket$. Let $U \subseteq \llbracket \circ \varphi \rightarrow \varphi \rrbracket$ be an $S$-invariant neighbourhood of $x$. Let $n$ be least so that $S^{n}(x) \in \llbracket \varphi \rrbracket$; if $n>0$, since $U$ is $S$-invariant we see that $S^{n-1}(x) \in U \subseteq \llbracket \circ \varphi \rightarrow \varphi \rrbracket$, hence $S^{n-1}(x) \in \llbracket \varphi \rrbracket$, contradicting the minimality of $n$. Thus $n=0$ and $x \in \llbracket \varphi \rrbracket$.

The additional axioms we have considered are valid over specific classes of dynamical systems. Specifically, the constant domain axiom is valid for the class of dynamic posets, while the Fischer Servi axioms are valid for the class of open systems. Let us begin by discussing the former in more detail.

Theorem 2. ITL ${ }^{\mathrm{CD}}$ and $\mathrm{ITL} \mathrm{L}_{\square}^{\mathrm{CD}}$ are sound for the class of dynamic posets; that is, $\mathrm{ITL}^{\mathrm{CD}} \subseteq \mathrm{ITL}^{\mathrm{e}}$ and $\mathrm{ITL} \mathrm{C}_{\square}^{\mathrm{CD}} \subseteq \mathrm{ITL} \mathrm{L}_{\square}^{\mathrm{e}}$.

Proof. Let $\mathcal{M}=(X, \preccurlyeq, S, \llbracket \cdot \rrbracket)$ be a dynamic poset model; in view of Theorem 1 . it only remains to check that $\mathrm{CD}$ and $\mathrm{BI}$ are valid on $\mathcal{M}$. However, by Proposition 1. BI is a consequence of CD, so we only check the latter. Suppose that $x \in \llbracket \square(\varphi \vee \psi) \rrbracket$, but $x \notin \llbracket \square \varphi \rrbracket$. Then, in view of Lemma 2, for some $n \geq 0$, $S^{n}(x) \notin \llbracket \varphi \rrbracket$. It follows that $S^{n}(x) \in \llbracket \psi \rrbracket$, so that $x \in \llbracket \triangleright \psi \rrbracket$. 
Note that the relational semantics are used in an essential way, since Lemma 2 is not available in the topological setting, and indeed we will show in Proposition 4 that these axioms are not topologically valid. But before that, let's turn our attention to the Fischer Servi axioms.

Theorem 3. $\mathrm{ITL}^{\mathrm{FS}} \subseteq \mathrm{ITL}^{\circ}$, i.e. $\mathrm{ITL}^{\mathrm{FS}}$ is sound for the class of open dynamical systems.

Proof. Let $\mathcal{M}=(X, \mathcal{T}, S, \llbracket \cdot \rrbracket)$ be a dynamical topological model where $S$ is an interior map. We check that axioms $\mathrm{FS}_{\circ}$ and $\mathrm{FS}_{\diamond}$ are valid on $\mathcal{M}$.

$\left(\mathrm{FS}_{\circ}\right)$ Suppose that $x \in \llbracket \circ \varphi \rightarrow \circ \psi \rrbracket$, and let $U \subseteq \llbracket \circ \varphi \rightarrow \circ \psi \rrbracket$ be a neighbourhood of $x$. Since $S$ is open, $V=S[U]$ is a neighbourhood of $S(x)$. Let $y \in V \cap \llbracket \varphi \rrbracket$, and choose $z \in U$ so that $y=S(z)$. Then, $z \in U \cap \llbracket \circ \varphi \rrbracket$, so that $z \in \llbracket \circ \psi \rrbracket$, i.e. $y \in \llbracket \psi \rrbracket$. Since $y \in V$ was arbitrary, $S(x) \in \llbracket \varphi \rightarrow \psi \rrbracket$, and $x \in \llbracket \circ(\varphi \rightarrow \psi) \rrbracket$.

$\left(\mathrm{FS}_{\diamond}\right)$ Suppose that $x \in \llbracket \diamond \varphi \rightarrow \square \psi \rrbracket$, and let $U \subseteq \llbracket \diamond \varphi \rightarrow \square \psi \rrbracket$ be a neighbourhood of $x$. Set $V=\bigcup_{n<\omega} S^{n}[U]$; since $S$ is open and unions of opens are open, $V$ is open as well. Moreover, $V$ is clearly $S$-invariant, as if $x \in V$, then $x \in S^{n}[U]$ for some $n \geq 0$, so that $S(x) \in S^{n+1}[U] \subseteq V$.

We claim that $V \subseteq \llbracket \varphi \rightarrow \psi \rrbracket$, from which we obtain a witness that $\mathcal{M}, x \models$ $\square(\varphi \rightarrow \psi)$. Suppose that $y \in V \cap \llbracket \varphi \rrbracket$. By the definition of $V, y=S^{n}(z)$ for some $n<\omega$ and some $z \in U$. Then, $z \in U \cap \llbracket \nabla \varphi \rrbracket$, so that $z \in \llbracket \square \psi \rrbracket$. From this we may choose an $S$-invariant neighbourhood $Z \subseteq \llbracket \psi \rrbracket$ of $z$. But $y=S^{n}(z) \in Z$ so that $y \in \llbracket \psi \rrbracket$, and since $y \in V$ was arbitrary we see that $V \subseteq \llbracket \varphi \rightarrow \psi \rrbracket$, as needed.

As an easy consequence, we mention the following combination of Theorems 2 and 3. Recall that dynamic posets with an interior map are also called persistent.

Corollary 1. $\mathrm{ITL}^{1}$ and $\mathrm{ITL}_{\square}^{1}$ are sound for the class of persistent dynamic posets, that is, $\mathrm{ITL}^{1} \subseteq \mathrm{ITL}^{\mathrm{p}}$ and $\mathrm{ITL}_{\square}^{1} \subseteq \mathrm{ITL}_{\square}^{\mathrm{p}}$.

\section{Independence}

In this section we will use our soundness results to show that the four logics we have considered are pairwise distinct. First we note that the formulas CD and BI separate Kripke semantics from the general topological semantics.

Proposition 4. The formulas $\mathrm{CD}(p, q)$ and $\mathrm{BI}(p, q)$ are not valid over the class of invertible dynamical systems based on $\mathbb{R}$, hence ITL $\mathrm{FS}^{\mathrm{F}} \forall \mathrm{CD}(p, q)$ and $\mathrm{ITL}^{\mathrm{FS}} \forall$ $\mathrm{BI}(p, q)$.

Proof. Define a model $\mathcal{M}$ on $\mathbb{R}$, with $S(x)=2 x, \llbracket p \rrbracket=(-\infty, 1)$ and $\llbracket q \rrbracket=(0, \infty)$. Clearly $\llbracket p \vee q \rrbracket=\mathbb{R}$, so that $\llbracket \square(p \vee q) \rrbracket=\mathbb{R}$ as well.

Let us see that $\mathcal{M}, 0 \not \models \mathrm{CD}(p, q)$. Since $\mathcal{M}, 0 \models \square(p \vee q)$, it suffices to show that $\mathcal{M}, 0 \forall \forall \square p \vee \diamond q$. It is clear that $\mathcal{M}, 0 \not \forall \diamond q$ simply because $S^{n}(0)=0 \notin \llbracket q \rrbracket$ 
for all $n$. Meanwhile, by Example $1, \mathcal{M}, 0=\square p$ if and only if $\llbracket p \rrbracket=\mathbb{R}$, which is not the case. We conclude that $\mathcal{M}, 0 \not \models \mathrm{CD}(p, q)$.

To see that $\mathcal{M}, 0 \not \models \mathrm{BI}(p, q)$ we proceed similarly, where the only new ingredient is the observation that $\mathcal{M}, 0 \models \square(\circ q \rightarrow q)$. But this follows easily from the fact that if $\mathcal{M}, x \models \circ q$, then $x>0$ so that $\mathcal{M}, x \models q$, hence $\llbracket \circ q \rightarrow q \rrbracket=\mathbb{R}$.

Proposition 5. The formula $\mathrm{BI}(p, q) \rightarrow \mathrm{CD}(p, q)$ is not valid over the class of invertible dynamical systems based on $\mathbb{R}$.

Proof. Consider a model $\mathcal{M}$ similar to that used in the proof of Proposition 4 , except that $\llbracket q \rrbracket=\mathbb{R} \backslash[-1 / 2,1 / 2]$. Then, $\square(p \vee q) \rightarrow \square p \vee \diamond q$ fails at 0 (by essentially the same reasoning). However, it could easily be checked that $\llbracket \square(\circ q \rightarrow q) \rrbracket=\llbracket q \rrbracket$. Hence $0 \in \llbracket \neg \square(\circ q \rightarrow q) \rrbracket$, from which it readily follows that 0 satisfies

$$
\square(\circ q \rightarrow q) \rightarrow(\square(p \vee q) \rightarrow \square p \vee q) .
$$

Therefore $\mathrm{BI}(p, q)$ does not imply $\mathrm{CD}(p, q)$ over the class of invertible dynamical systems.

Note, however, that Proposition 5 does not necessarily imply that there are no formulas $\varphi, \psi$ such that $\operatorname{BI}(\varphi, \psi) \rightarrow \operatorname{CD}(p, q)$ is derivable, and hence it is reasonable to use $\mathrm{BI}$ in place of $\mathrm{CD}$ to axiomatize $\diamond$-free logics.

The Fischer Servi axioms are also not valid in general, as shown in Boudou et al. 2. From this and the soundness of ITL ${ }^{\mathrm{FS}}$ (Theorem 3), we immediately obtain that they are not derivable in $\mathrm{ITL}^{0}$.

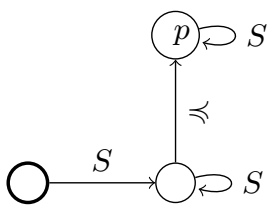

Fig. 2: A dynamic poset model falsifying both Fischer Servi axioms. Propositional variables that are true on a point are displayed; only one point satisfies $p$ and no point satisfies $q$. It can readily be checked that $\operatorname{FS}_{\diamond}(p, q)$ and $\mathrm{FS}_{\diamond}(p, q)$ fail on the highlighted point on the left. Note that $S$ is continuous but not open, as can easily be seen by comparing to Figure 1 .

Proposition 6. $\mathrm{FS}_{\circ}(p, q)$ and $\mathrm{FS}_{\diamond}(p, q)$ are not valid over the class of dynamic posets, hence $\mathrm{ITL}^{\mathrm{CD}} \not \mathrm{FS}_{\diamond}(p, q)$ and $\mathrm{ITL}^{\mathrm{CD}} \not \mathrm{FS}_{\diamond}(p, q)$.

Proof. Let $\{p, q\}$ be a set of propositional variables and let us consider the model $\mathcal{M}=(W, \preccurlyeq, S, V)$ defined by 1) $W=\{w, v, u\}$; 2) $S(w)=v, S(v)=v$ and $S(u)=u$;3) $v \preccurlyeq u$; 4) $V(p)=\{u\}$, and 5) $V(q)=\varnothing$ (see Figure 2). Clearly, $\mathcal{M}, u \not \models p \rightarrow q$, so $\mathcal{M}, v \not \models p \rightarrow q$. By definition, $\mathcal{M}, w \not \models \circ(p \rightarrow q)$ and 
$\mathcal{M}, w \not \models \square(p \rightarrow q)$; however, $\mathcal{M}, w \models \circ p \rightarrow \circ q$ and $\mathcal{M}, w \models \diamond p \rightarrow \square q$ since the negation of each antecedent holds, so $\mathcal{M}, w \not \models(\circ p \rightarrow \circ q) \rightarrow \circ(p \rightarrow q)$ and $\mathcal{M}, w \not \models(\diamond p \rightarrow \square q) \rightarrow \square(p \rightarrow q)$.

Remark 2. As mentioned previously, Yuse and Igarashi [29] present a Hilbertcalculus which yields a sub-logic of $I T L_{\square}^{0}$. They also present a Gentzen-style calculus and conjecture that their two calculi prove the same set of formulas. However, Kojima and Igarashi [19] show that the formula $\mathrm{FS}_{\circ}(p, q)$ is derivable in this Gentzen calculus. Thus Proposition 6 shows that the two calculi are not equivalent.

The above independence results are sufficient to see that each of our four syntactically-defined logics, as well as each of our four semantically-defined logics, are pairwise distinct.

Theorem 4. The logics $\mathrm{ITL}^{0}, \mathrm{ITL}^{\mathrm{FS}}$, ITL ${ }^{\mathrm{CD}}$ and $\mathrm{ITL}^{1}$ are pairwise distinct, as are $\mathrm{ITL}_{\square}^{0}$, ITL $\mathrm{IL}_{\square}^{\mathrm{FS}}$, ITL $\mathrm{IL}_{\square}^{\mathrm{CD}}$ and $\mathrm{ITL} \mathrm{L}_{\square}^{1}$. Similarly, $\mathrm{ITL}^{\mathrm{c}}$, ITL $\mathrm{IT}^{\circ}, \mathrm{ITL}^{\mathrm{e}}$ and $\mathrm{ITL}^{\mathrm{p}}$ are pairwise distinct, as are $\mathrm{ITL} \mathrm{L}_{\square}^{\mathrm{c}}$, $\mathrm{ITL} \mathrm{\circ}_{\square}^{\circ}$, $\mathrm{ITL} \mathrm{L}_{\square}^{\mathrm{e}}$ and $\mathrm{ITL}_{\square}^{\mathrm{p}}$.

Proof. By Proposition 4 and the definition of $\mathrm{ITL}^{\mathrm{CD}}, \mathrm{CD}(p, q) \in \mathrm{ITL}^{\mathrm{CD}} \backslash \mathrm{ITL}^{\mathrm{FS}}$; similarly, by Proposition $6, \mathrm{FS}_{\circ}(p, q) \in \mathrm{ITL}^{\mathrm{FS}} \backslash \mathrm{ITL}^{\mathrm{CD}}$. Thus $\mathrm{ITL}^{\mathrm{FS}}$ and $\mathrm{ITL}^{\mathrm{CD}}$ are incomparable, from which we conclude that $\mathrm{ITL}^{0}$, which is contained in their intersection, is strictly smaller than either of them, while $\mathrm{ITL}^{1}$, which contains their union, is strictly larger. The arguments for the logics over $\mathcal{L}_{\square}$ are analogous, except that CD is replaced with BI, as is the argument for semantically-defined logics.

\section{Concluding Remarks}

We have proposed a natural 'minimalist' intuitionistic temporal logic, $\mathrm{ITL}^{0}$, along with possible extensions including Fischer Servi or constant domain axioms. We have seen that relational semantics validate the constant domain axiom, leading us to consider a wider class of models based on topological spaces, with a novel interpretation for 'henceforth' based on invariant neighbourhoods. With this, we have shown that the logics ITL ${ }^{0}, I_{T L}{ }^{C D}, I T L^{F S}$ and $I T L^{1}$ are sound for the class of all dynamical systems, of all dynamical posets, of all open dynamical systems, and of all persistent dynamical posets, respectively, which we have used in order to prove that the logics are pairwise distinct. Of course this immediately raises the question of completeness, which we have not addressed. Specifically, the following are left open.

Question 1. Are the logics:

- ITL ${ }^{0}$ and $\mathrm{ITL}_{\square}^{0}$ complete for the class of dynamical systems?

- ITL ${ }^{\mathrm{CD}}$ and ITL $\mathrm{L}_{\square}^{\mathrm{CD}}$ complete for the class of dynamic posets?

- ITL ${ }^{\mathrm{FS}}, \mathrm{ITL}_{\diamond}^{\mathrm{FS}}$ and $\mathrm{ITL}_{\square}^{\mathrm{FS}}$ complete for the class of open dynamical systems?

- ITL ${ }^{1}, \mathrm{ITL}_{\diamond}^{1}$ and $\mathrm{ITL}_{\square}^{1}$ complete for the class of persistent dynamic posets? 
We already know that $\mathrm{ITL}_{\diamond}^{0}$ is sound and complete for the class of dynamic posets [12]. However, the completeness of $\mathrm{ITL}_{\diamond}^{\mathrm{FS}}$ and $\mathrm{ITL}_{\diamond}^{1}$ is likely to be a more difficult problem than that of $\mathrm{ITL}_{\diamond}^{0}$, as in these cases it is not even known if the set of valid formulas is computably enumerable, let alone decidable.

Question 2. Are any of the logics $\Lambda, \Lambda_{\diamond}$, or $\Lambda_{\square}$ with $\Lambda \in\left\{\mathrm{ITL}^{\mathrm{p}}, \mathrm{ITL}^{\circ}\right\}$ decidable and/or computably enumerable?

In any of these cases a negative answer is possible, since that is the case for their classical counterparts [20] and these logics do not have the finite model property [4. Nevertheless, the proofs of non-axiomatizability in the classical case do not carry over to the intuitionistic setting in an obvious way, and these remain challenging open problems.

\section{References}

1. Aleksandroff, P.: Diskrete räume. Matematicheskii Sbornik 2(44), 501-518 (1937)

2. Balbiani, P., Boudou, J., Diéguez, M., Fernández-Duque, D.: Bisimulations for intuitionistic temporal logics. arXiv 1803.05078 (2018)

3. Balbiani, P., Diéguez, M.: Temporal here and there. In: Loizos, M., Kakas, A. (eds.) Logics in Artificial Intelligence. pp. 81-96. Springer (2016)

4. Boudou, J., Diéguez, M., Fernández-Duque, D.: A decidable intuitionistic temporal logic. In: 26th EACSL Annual Conference on Computer Science Logic (CSL). vol. 82, pp. 14:1-14:17 (2017)

5. Brewka, G., Eiter, T., Truszczyński, M.: Answer set programming at a glance. Communications of the ACM 54(12), 92-103 (2011)

6. Cabalar, P., Pérez Vega, G.: Temporal equilibrium logic: A first approach. In: Computer Aided Systems Theory - EUROCAST'07. pp. 241-248. Springer Berlin Heidelberg (2007)

7. Davies, R.: A temporal-logic approach to binding-time analysis. In: Proceedings, 11th Annual IEEE Symposium on Logic in Computer Science, New Brunswick, New Jersey, USA, July 27-30, 1996. pp. 184-195 (1996)

8. Davies, R.: A temporal logic approach to binding-time analysis. Journal of the ACM 64 (2017)

9. Davies, R., Pfenning, F.: A modal analysis of staged computation. J. ACM 48(3), 555-604 (2001)

10. Davoren, J.M.: On intuitionistic modal and tense logics and their classical companion logics: Topological semantics and bisimulations. Annals of Pure and Applied Logic 161(3), 349-367 (2009)

11. Davoren, J.M., Coulthard, V., Moor, T., Gor, R., Nerode, A.: Topological semantics for intuitionistic modal logics, and spatial discretisation by A/D maps. In: Workshop on Intuitionistic Modal Logic and Applications (IMLA) (2002)

12. Diéguez, M., Fernández-Duque, D.: An intuitionistic axiomatization of 'eventually'. In: Advances in Modal Logic. vol. 12, pp. 199-218 (2018)

13. Ershov, A.P.: On the partial computation principle. Inf. Process. Lett. 6(2), 38-41 (1977)

14. Fernández-Duque, D.: The intuitionistic temporal logic of dynamical systems. Logical Methods in Computer Science 14(3) (2018) 
15. Fischer Servi, G.: Axiomatisations for some intuitionistic modal logics. In: Rendiconti del Seminario Matematico. vol. 42, pp. 179-194. Universitie Politecnico Torino (1984)

16. Goldblatt, R.: Logics of Time and Computation. No. 7 in CSLI Lecture Notes, Center for the Study of Language and Information, Stanford, CA, 2 edn. (1992)

17. Howard, W.A.: The formulas-as-types notion of construction. In: Seldin, J.P., Hindley, J.R. (eds.) To H. B. Curry: Essays on Combinatory Logic, Lambda Calculus, and Formalism, pp. 479-490. Academic Press (1980)

18. Kamide, N., Wansing, H.: Combining linear-time temporal logic with constructiveness and paraconsistency. Journal of Applied Logic 8(1), 33-61 (2010)

19. Kojima, K., Igarashi, A.: Constructive linear-time temporal logic: Proof systems and Kripke semantics. Information and Computation 209(12), 1491 -1503 (2011)

20. Konev, B., Kontchakov, R., Wolter, F., Zakharyaschev, M.: On dynamic topological and metric logics. Studia Logica 84, 129-160 (2006)

21. Kremer, P.: A small counterexample in intuitionistic dynamic topological logic. http://individual.utoronto.ca/philipkremer/onlinepapers/counterex.pdf (2004)

22. Lichtenstein, O., Pnueli, A.: Propositional temporal logics: Decidability and completeness. Logic Jounal of the IGPL 8(1), 55-85 (2000)

23. Maier, P.: Intuitionistic LTL and a new characterization of safety and liveness. In: Marcinkowski, J., Tarlecki, A. (eds.) 18th EACSL Annual Conference on Computer Science Logic (CSL). pp. 295-309. Springer Berlin Heidelberg, Berlin, Heidelberg (2004)

24. Mints, G.: A Short Introduction to Intuitionistic Logic. University Series in Mathematics, Springer (2000)

25. Munkres, J.R.: Topology. Featured Titles for Topology Series, Prentice Hall, Incorporated (2000)

26. Pacuit, E.: Neighborhood Semantics for Modal Logic. Springer (2017)

27. Pnueli, A.: The temporal logic of programs. In: 18th Annual Symposium on Foundations of Computer Science (sfcs 1977). pp. 46-57 (1977)

28. Simpson, A.K.: The proof theory and semantics of intuitionistic modal logic. Ph.D. thesis, University of Edinburgh, UK (1994)

29. Yuse, Y., Igarashi, A.: A modal type system for multi-level generating extensions with persistent code. In: Proceedings of the 8th ACM SIGPLAN International Conference on Principles and Practice of Declarative Programming. pp. 201-212. PPDP '06 (2006) 